\title{
Guest Editors' Introduction to the Special Section on the Impact of COVID-19 on Sexual Health and Behaviors
}

\author{
Lori A. J. Scott-Sheldon ${ }^{1} \cdot$ Kristen P. Mark ${ }^{2} \cdot$ Rhonda N. Balzarini ${ }^{3,4} \cdot$ Lisa L. M. Welling ${ }^{5}$
}

Published online: 28 January 2022

This is a U.S. government work and not under copyright protection in the U.S.; foreign copyright protection may apply 2022

The World Health Organization officially declared the novel coronavirus disease (COVID-19) a pandemic on March 11, 2020 (World Health Organization [WHO], 2020). In a short span of time, the COVID-19 pandemic mitigation measures caused widespread social and economic disruptions to individuals, communities, and nations. Many of the early mitigation measures have since been lifted, but the persistent threat of SARS-CoV-2, the virus that causes COVID-19, and its variants of concern, and the slow rate of vaccination in the USA and worldwide, threatens the health, safety, and well-being of all individuals. To call attention to the global impact of the pandemic on sexual health and behaviors, including sexual relationships, health care access and treatment, and sexual and reproductive rights, we invited a broad range of sexual health scholars to contribute to this Special Section. The global impact of COVID-19 on sexual health and behaviors is beginning to emerge, as highlighted in this issue, showing how some groups may be more vulnerable to the pandemic.

The first set of papers examine the influence of the COVID-19 pandemic on sex and sexuality. Maes and Vandenbosch (2022) investigated changes in adolescents' use of sexually explicit Internet material before, during, and after a strict lockdown period was established in Belgium. Although reported use of sexually explicit material did not increase significantly across the 15-month study period, girls showed a greater increase in the use

Lori A. J. Scott-Sheldon

lori.scott-sheldon@nih.gov

1 Division of AIDS Research, National Institute of Mental Health, 5601 Fishers Lane, Bethesda, MD 20892, USA

2 Department of Family Medicine and Community Health, University of Minnesota Medical School, Minneapolis, MN, USA

3 Department of Psychology, Texas State University, San Marcos, TX, USA

4 Kinsey Institute, Indiana University, Bloomington, IN, USA

5 Department of Psychology, Oakland University, Rochester, MI, USA of sexually explicit Internet material relative to boys. Likewise, as part of an ongoing study on sexual health, Bőthe et al. (2022) documented potential changes in Canadian adolescents' pornography use frequency, motivations, and problematic pornography use before and during the COVID-19 pandemic. They found no significant changes in adolescents' pornography use frequency or motivations, and only very minor changes in problematic pornography use. In a similar study involving longitudinal data collected from a large sample of American adults, Grubbs et al. (2021) explored pornography use before and during the COVID19 pandemic. Even though pornography use was highest during the height of the first-wave lockdown period than at any other follow-up time, reported use was still lower than self-reported use at baseline and pornography use trended downward for both men and women over time. Together, these results suggest that the COVID-19 pandemic has had a marginal or no real lasting impact on the use of pornographic materials, but the next two papers highlight the relevance of the COVID-19 pandemic to sexual psychology. Thomas et al. (2022) administered two online surveys, conducted during the strict federal lockdown, to investigate the effects of social isolation on willingness to engage in sexting among Austrian adults. Results from these surveys showed that the more socially isolated people felt during lockdown, the more they were willing to engage in sexting, suggesting that sexting may have been used as a coping mechanism during the early days of the COVID-19 pandemic. Nobre et al. (2022) used a longitudinal design to examine how personality and psychosexual factors predict sexual functioning and sexual distress among Portuguese adults during and following the COVID-19 mitigation phase (i.e, lockdown). Interestingly, sexual distress decreased post-lockdown in both men and women but, among men only, so too did sexual functioning.

The next set of papers examined the influence of the COVID19 pandemic on sexual relationships. Herbenick et al. (2022) conducted an online survey of college students at a large Midwestern university in the USA both before and after campus closed due to the pandemic. They found many sexual behaviors remained unchanged but participation in oral, vaginal, and anal 
sex significantly decreased over time. Additionally, when partnered sexual behavior did take place, it was more often with a cohabiting relationship partner and was rated as more wanted, emotionally intimate, and orgasmic. Hicks et al. (2021) used daily diary methodology across a 2 -week period to examine concerns of contracting COVID-19. They found that people with higher perceived infectability enjoyed sex less on days that they were more worried about contracting COVID-19 than usual. Related to perception of infectability, Price et al. (2021) collected data from American men on their COVID-19 risk perception. Gay and bisexual men were no different than heterosexual men in their level of risk perception, the extent to which they engaged in social distancing, or risk perceptions of transmission through sex outside of their social grouping. Gay and bisexual men were over twice as likely than heterosexual men to follow Centers for Disease Control and Prevention recommended precautions and held higher risk perceptions of intimate activities despite engaging in more sex and more socializing outside of their social group.

Also examining sexual minority men, Starks et al. (2021) used Interdependence Theory, a framework for understanding how relationship partners influence health behaviors, and found that joint prevention efforts between romantic partners was positively related to COVID-19 prevention behaviors. They also found that the more satisfied a couple is, the more likely they engaged in a collaborative approach to prevention, emphasizing the importance of relationships in prevention of COVID19 spread. Stulhofer et al. (2022) found that a little over half of participants reported no change in their sexual interest during the pandemic and this was similar across seven European Union countries and Turkey. Additionally, this study found that distress around desire discrepancy between partners was associated with coping with and worrying about the pandemic, relationship quality and emotional closeness, and motivation for sex. Pollard and Rogge (2022) used a Family Systems Theory framework to demonstrate that COVID-19-related stress corresponded to poorer sexual functioning, which led to decreased relationship functioning and overall individual well-being. The final two papers in this set examined sexual relationship health and adjustment during COVID-19. Gauvin et al. (2022) concluded that people in relationships were relatively resilient to the COVID-19 pandemic, with only slight declines in sexual pleasure, frequency of orgasms with a partner, and frequency of solitary orgasms compared to retrospective recall of pre-pandemic levels. Finally, Griffin et al. (2022) found that self-reported sexual activity and the number of sexual partners decreased during the first wave of the pandemic in a sample 1051 LGBTQ+adults in the USA, but sexual activity was impacted by living arrangement and partner status.

The final set of papers addressed the profound impact of the COVID-19 pandemic on sexual healthcare, prevention, and treatment in the USA, Canada, and India. Zapata et al. (2021) conducted online focus groups with young sexual minority men (YSMM) living in the USA to explore the impact of the pandemic on HIV testing and prevention services. YSMM identified challenges to accessing and receiving HIV testing services, preexposure prophylaxis (PrEP), and HIV post-exposure prophylaxis (PEP) during the pandemic, which was exacerbated by anticipated rejection from their families, as many YSMM had moved back home during the early months of the pandemic, concerns about placing their families at risk for COVID-19 by attending clinic appointments, and lack of appropriate services for YSMM. Similarly, Parikh et al. (2022) identified barriers to accessing sexual and reproductive health (SRH) services among people living with HIV (PLHIV) across five states in India. Qualitative interviews with PLHIV and their health care providers highlighted the profound disruptions in SRH services during the pandemic including restricted access to clinics, limited availability of antiretroviral therapy, and financial strains due to shortages in staffing, medications, supplies, and employment loss. Benoit and Unsworth (2021) examined how persistent stigma affected sex workers during the COVID-19 pandemic from the perspective of organizational support staff who provide critical health and social services to sex workers to increase our understanding of what it will take to avoid the economic, social, and health harms of COVID-19 among sex workers in Canada. The next two papers in this set focused on the effects of the pandemic on sexual behaviors, mental health, and preventive sexual health care highlighting the disruptions in sexual behavior (partner reduction), accessing and receiving PrEP and other HIV services, and pandemic-induced increases in anxiety and depression among sexual and gender minorities living in the USA (Grov et al., 2021) and the sudden and pronounced increase in depression and anxiety following the onset of the COVID-19 pandemic in an ongoing cohort of Australian gay and bisexual men (Bavinton et al., 2022). The last paper in this set describes the increased vigilance in the use of pregnancy prevention methods (e.g., intrauterine device, oral contraceptives, implants) among US adults during the pandemic (Fikslin et al., 2021).

The papers included in this Special Section represent a broad range of studies on sexual health and behaviors conducted before, during, and after the onset of the COVID-19 pandemic. While this Special Section highlights the early impact of COVID-19 on sexual health and behaviors, more research is needed to understand the ongoing, and potentially long-term, effects of the pandemic on sexual health and behaviors, including the development, testing, and implementation of effective strategies to provide optimal sexual healthcare while simultaneously reducing the risk of acquiring SARS-CoV-2. These papers, taken together, describe the widespread impact of COVID-19 on sexuality, sexual relationships, sexual healthcare, prevention, and treatment particularly among marginalized groups and highlight the need for concerted public health efforts to support sexual health in the era of COVID-19 and beyond.

Acknowledgements The views expressed in this article do not necessarily represent the views of the National Institutes of Health, the 
Department of Health and Human Services, or the United States Government. We thank the Editor for his assistance with this Special Section on the Impact of COVID-19 on Sexual Health and Behaviors.

\section{References}

Bavinton, B. R., Chan, C., Hammoud, M. A., Maher, L., Haire, B., Degenhardt, L., \& Prestage, G. P. (2022). Increase in depression and anxiety among Australian gay and bisexual men during COVID-19 restrictions: Findings from a prospective online cohort study. Archives of Sexual Behavior. https://doi.org/10.1007/ s10508-021-02276-2

Benoit, C., \& Unsworth, R. (2021). COVID-19, stigma, and the ongoing marginalization of sex workers and their support organizations. Archives of Sexual Behavior. https://doi.org/10.1007/ s10508-021-02124-3

Bőthe, B., Vaillancourt-Morel, M.-P., Dion, J., Paquette, M.-M., Massé-Pfister, M., Tóth-Király, I., \& Bergeron, S. (2022). A longitudinal study of adolescents' pornography use frequency, motivations, and problematic use before and during the COVID-19 pandemic. Archives of Sexual Behavior. https://doi.org/10.1007/ s10508-021-02282-4

Fikslin, R. A., Goldberg, A. J., Gesselman, A. N., Reinka, M. A., Pervez, O., Franklin, E. T., \& Price, D. M. (2021). Changes in utilization of birth control and PrEP during COVID-19 in the U.S.: A mixedmethod analysis. Archives of Sexual Behavior. https://doi.org/10. 1007/s10508-021-02086-6

Gauvin, S. E. M., Mulroy, M. E., McInnis, M. K., Jackowich, R. A., Levang, S. L., Coyle, S. M., \& Pukall, C. F. (2022). An investigation of sexual and relationship adjustment during COVID-19. Archives of Sexual Behavior. https://doi.org/10.1007/s10508-021-02212-4

Griffin, M., Jaiswal, J., Martino, R. J., LoSchiavo, C., Comer-Carruthers, C., Krause, K. D., \& Halkitis, P. N. (2022). Sex in the time of COVID-19: Patterns of sexual behavior among LGBTQ+ individuals in the United States. Archives of Sexual Behavior. https:// doi.org/10.1007/s10508-022-02298-4

Grov, C., Zohra, F., Westmoreland, D. A., Mirzayi, C., D’Angelo, A., Stief, M., \& Carrico, A. W. (2021). Sex in the era of COVID19 in a U.S. national cohort of cisgender men, transgender women, and transgender men who have sex with men: AprilMay 2020. Archives of Sexual Behavior. https://doi.org/10.1007/ s10508-021-02121-6

Grubbs, J. B., Perry, S. L., Weinandy, J. T., \& Kraus, S. W. (2021). Porndemic? A longitudinal study of pornography use before and during the COVID-19 pandemic in a nationally representative sample of Americans. Archives of Sexual Behavior. https://doi.org/10.1007/ s10508-021-02077-7

Herbenick, D., Hensel, D. J., Eastman-Mueller, H., Beckmeyer, J., Tsung-Chieh, F., Guerra-Reyes, L., \& Rosenberg, M. (2022). Sex and relationships pre- and early- COVID-19 pandemic: Findings from a probability sample of U.S. undergraduate students. Archives of Sexual Behavior. https://doi.org/10.1007/s10508-021-02265-5

Hicks, L. L., Meltzer, A. L., French, J. E., Altgelt, E. E., Turner, J. A., \& McNulty, J. K. (2021). Perceptions of infectability to disease moderate the association between daily concerns about contracting COVID-19 and statisfaction with sex. Archives of Sexual Behavior. https://doi.org/10.1007/s10508-021-02076-8
Maes, C., \& Vandenbosch, L. (2022). Adolescents' use of sexually explicit internet material over the course of 2019-2020 in the context of the COVID-19 pandemic: A three-wave panel study. Archives of Sexual Behavior. https://doi.org/10.1007/ s10508-021-02122-5

Nobre, P., Rosa, P. J., Vasconcelos, P., Tavares, I., Carvalho, J., QuntaGomes, A., \& Carrito, M. (2022). Sexual health and the pandemic crisis: Testing the role of psychological vulnerability/protective factors on sexual functioning and sexual distress during a critical life period in Portugal. Archives of Sexual Behavior. https://doi. org/10.1007/s10508-021-02209-z

Parikh, N., Chaudhuri, A., Syam, S. B., Singh, P., Pal, P., \& Pillala, P. (2022). Diseases and disparities: The impact of COVID-19 disruptions on sexual and reproductive health services among the HIV community in India. Archives of Sexual Behavior. https://doi.org/ 10.1007/s10508-021-02211-5

Pollard, A. E., \& Rogge, R. D. (2022). Love in the time of COVID-19: A multi-wave study examining the salience of sexual and relationship health during the COVID-19 pandemic. Archives of Sexual Behavior. https://doi.org/10.1007/s10508-021-02208-0

Price, D. M., Gesselman, A. N., Fikslin, R. A., Goldberg, A. J., Pervez, O., Reinka, M. A., \& Franlin, E. (2021). How can I get COVID? Understanding differences in American heterosexual and sexual minority men's risk perception. Archives of Sexual Behavior. https://doi.org/10.1007/s10508-021-02123-4

Starks, T. J., Bosco, S. C., Doyle, K. M., \& Revenson, T. A. (2021). Partners' consensus about joint effort and COVID-19 prevention among sexual minority men. Archives of Sexual Behavior. https:// doi.org/10.1007/s10508-021-02063-z

Štulhofer, A., Mehulić, J., Briken, P., Klapilová, K., de Graaf, H., Carvalheira, A. A., \& Schröder, J. (2022). Perceived changes in sexual interest and distress about discrepant sexual interest during the first phase of COVID-19 pandemic: A multi-country assessment in cohabiting partnered individuals. Archives of Sexual Behavior. https://doi.org/10.1007/s10508-021-02279-z

Thomas, M. F., Binder, A., \& Matthes, J. (2022). Love in the time of Corona: Predicting willingness to engage in sexting during the COVID-19-related lockdown. Archives of Sexual Behavior. https:// doi.org/10.1007/s10508-022-02292-w

World Health Organization. (2020). WHO Director-General's opening remarks at the media briefing on COVID-19. Retrieved from https://www.who.int/director-general/speeches/detail/ who-director-general-s-opening-remarks-at-the-media-brief ing-on-covid-19---11-march-2020.

Zapata, J. P., Dang, M., Quinn, K. G., Horvath, K. J., Stephenson, R., Dickson-Gomez, J., \& John, S. A. (2021). COVID-19-related disruptions to HIV testing and prevention among young sexual minority men 17-24 years old: A qualitative study using synchronous online focus groups, April-September 2020. Archives of Sexual Behavior. https://doi.org/10.1007/s10508-021-02166-7

Publisher's Note Springer Nature remains neutral with regard to jurisdictional claims in published maps and institutional affiliations. 